\title{
Activity dependent modulation of synaptic transmission by presynaptic calcium stores: A dichotomy of short-term depression and facilitation
}

\author{
Suhita Nadkarni ${ }^{{ }^{*}}$, Thomas Bartol ${ }^{2}$, Herbert Levine ${ }^{3}$, Terrence Sejnowski ${ }^{2}$ \\ From Twenty Second Annual Computational Neuroscience Meeting: CNS*2013 \\ Paris, France. 13-18 July 2013
}

Ongoing electrical activity can initiate a positive feedback loop via Inositol Triphosphate Receptors $\left(\mathrm{IP}_{3} \mathrm{Rs}\right)$ and Ryanodine Receptors (RyRs) on the Endoplasmic Receptors (ER) that can lead to release of calcium from the ER. We investigated how the presence of this additional source of calcium in the presynaptic terminals in addition to the Voltage Dependent Calcium Channels (VDCCs) can regulate synaptic transmission. We carried out 3D Monte Carlo simulations of all the molecular interactions that govern transmitter release in a 1) Canonical CA3-CA1 synapse 2) A synapse reconstructed from serial section Transmission Electron Microscope images. The relatively simple geometry of CA3-CA1 synapses allows activity-dependent local calcium at the active zone and the related transmitter release profiles to be quantitatively analyzed. In paired-pulse stimulation, the presence of the molecular pathways that regulate the calcium stores increased the calcium buffering capacity of the synapse, which decreased release probability and enhanced paired-pulse facilitation. In contrast, a high-frequency stimulus could trigger the activation of presynaptic RyRs and Metabotropic Glutamate Receptors (mGluRs) leading to $\mathrm{IP}_{3}$ production and ultimately to release of calcium from the SER. $\mathrm{IP}_{3} \mathrm{Rs}$ and RyRs operated at a much slower time scale, on the order of seconds compared to the millisecond timescale of the VDCCs. This led to an increase in the basal level of intracellular calcium and enhanced transmitter release rates. In reconstructions of hippocampal neuropil, the
ER appears in a majority of the presynaptic terminals in varying degrees of abundance. We further explored the functional implications of the range of ER geometries and therefore the calcium carrying capacity, observed in the synaptic ultrastructure and the effect of different arrangements between $\mathrm{IP}_{3} R s$ and VDCCs on synaptic plasticity. The synaptic ultrastructure precisely orchestrated the degree of facilitation and depression at the synapse and the existence of presynaptic calcium stores provided the synapse with an additional intrinsic time scale that could be regulated by activity.

\section{Acknowledgements}

This work has been supported by Wellcome-Trust/DBT India Alliance grant to Suhita Nadkarni, NIH Grant R01MH079076, NSF sponsored Center for Theoretical Biological Physics, Grant PHY0216576 and PHY0225630, Howard Hughes Medical Institute HHMI.

\section{Author details}

'Wellcome Trust-DBT Intermediate Fellow Indian Institute of Science Education and Research, Pune, India. ${ }^{2}$ Salk Institute for Biological Studies, La Jolla, 92037, USA. ${ }^{3}$ Rice University, Houston Texas, 77030, USA.

Published: 8 July 2013

doi:10.1186/1471-2202-14-S1-P351

Cite this article as: Nadkarni et al:: Activity dependent modulation of synaptic transmission by presynaptic calcium stores: A dichotomy of short-term depression and facilitation. BMC Neuroscience 2013 14(Suppl 1): P351.

\footnotetext{
* Correspondence: suhita@iiserpune.ac.in

'Wellcome Trust-DBT Intermediate Fellow Indian Institute of Science

Education and Research, Pune, India

Full list of author information is available at the end of the article
} 